\section{International Scientific Journal} Theoretical \& Applied Science

p-ISSN: 2308-4944 (print) e-ISSN: 2409-0085 (online)

Year: $2016 \quad$ Issue: 4 Volume: 36

Published: $30.04 .2016 \quad$ http://T-Science.org
Manat Beyshenovich Tlebayev Doctor of engineering sciences, Professor, Academician of Kazakh National Academy of

Natural Sciences, Head of the department

"Computer engineering and Informatics"

Taraz State University named after M.Kh. Dulati,

Kazakhstan

Pavel Nikolayevich Shevtsov master student

Taraz State University named after M.Kh. Dulati,

Kazakhstan

pashka_111@bk.ru

\title{
COMPUTER MATHEMATICAL MODEL OF PHYSICO-MECHANICAL CHARACTERISTICS OF POLYMER COMPOSITES. DISCRETE CONTINUOUS PRESENTATION OF CARBON NANOMATERIALS
}

Abstract: The article presents a review of modern technologies of forming of composite materials with different fillers.

Key words: polyether matrix, carbon nanotubes, functionalization, composite material, nanocomposite, ultrasonic homogenization.

Language: Russian

Citation: Tlebayev MB, Shevtsov PN (2016) COMPUTER MATHEMATICAL MODEL OF PHYSICOMECHANICAL CHARACTERISTICS OF POLYMER COMPOSITES. DISCRETE CONTINUOUS PRESENTATION OF CARBON NANOMATERIALS. ISJ Theoretical \& Applied Science, 04 (36): $216-222$.

Soi: http://s-o-i.org/1.1/TAS-04-36-38 Doi: crossef http://dx.doi.org/10.15863/TAS.2016.04.36.38

\section{КОМПЬЮТЕРНО МАТЕМАТИЧЕСКАЯ МОДЕЛЬ ФИЗИКО-МЕХАНИЧЕСКИХ ХАРАКТЕРИСТИК ПОЛИМЕРНЫХ КОМПОЗИТОВ. ДИСКРЕТНО КОНТИНУАЛЬНАЯ ФОРМА ПРЕДСТАВЛЕНИЯ УГЛЕРОДНЫХ НАНОМАТЕРИАЛОВ}

Аннотация: В статье представлен обзор современных технологий формирования композиционных материалов с различными наполнителями.

Ключевые слова: полиэфирная матрица, углеродные нанотрубки, функциионализация, композитный материал, нанокомпозит, ультразвуковая гомогенизация.

Компьютерное моделирование и изучение проблем связанных с постройкой моделей материалов, описание их механических свойств, их структуры всегда были актуальными. Изучение и разборка всех проблем выявляет многие макроскопические зависимости характеристик деформирования структуры материала, прочностных характеристик и устойчивых или нет сопротивлений к разрушению.

Располагая всеми данными возможно расчетать параметрическую модель оптимизации при создании новых моделей материалов или всевозможных конструкций, а также испытательных характеристик.

В огромном многообразии новых материалов наиболее значимыми являются наноматериалы, обладающие наномасштабной структурой или же наполняемые наноструктурными частицами различных маштабов. Такие материалы обладают высокими значениями свойств деформирования, а также прочности и трещиностойкости. Обладатели таких характеристик применяются в промышленности.

Наноматериалы имеют широкий список вариаций, но выделяют углеродные наноматериалы, а в частности углеродные нанотрубки. Они выступают и в одиночном представлении в материале, также возможны варианты и в совокупности частиц или сплетений нановолокн в матрице других материалов.

Целью работ проводимых в этой области является создание модифицированных композитных материалов с введением нанотрубок и наночастиц в структуру матрицы. Изменения созданные таким образом, меняют в лучшую сторону характеристики продукта. 
Имеющийся литературный материал позволяет сделать вывод, что не значительные добавки наночастиц-фуллеренов, нанотрубок или нановолокн могут существенно, а временами даже в разы увеличить характеристики полимеров.

Одним из важнейших факторов является тот, что незначительные добавки УНТ(от одного до двух процентов, а иногда и на уровне 0,01-0,3 \%) увеличивают значения модуля упругости и разрывную прочность полимера в разы. в один момент увеличивается как теплопроводность так и электропроводность материала . Добавление углеродных трубок значительно расширяет диапазоны рабочих температур композитов на базе определенных полимеров вследствии повышения температуры перехода в стеклообразное состояние[1].

Рассмотрим дискренто-континуальные модели объектов, такие модели учитывают атомную структуру объектов. Так же приведем принципы построения таких моделей, их систем и отдельных нанообъектов. В рамках этой модели рассматривается и разрушение углеродных наноматериалов. Так же мы опишем алгоритм реализации моделей.

Применяя молекулярно динамическую методику возможно количественно описать ковалентные взаимодействия атомов молекулярной структуры. Зависимости от относительных атомных взаимодействий в каждой паре атомов зависят от сил притяжения и отталкивания и от относительного атомного положения, и описывается выбранным силовым полем. Такие силы осуществляют влияние на потенциальную колебательную энергию молекулярной системы. Данная энергия будет эквивалентна энергии деформации макроскопического тела схожего размера.

В гексагональной плоскости значимым элементом является четверка атомов представленная на рисунке ниже. В данной системе вклад э энергию деформации(растяжение, угловые изменения, не связанные взаимодействия, кручения) дают все степени свободы системы. Данные деформации представлены на рисунке 1 .

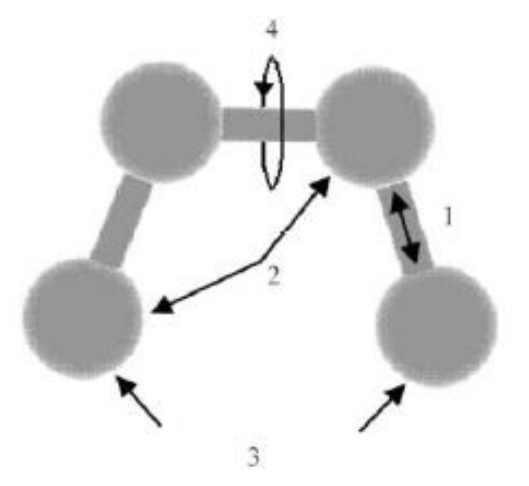

Рисунок 1 - Деформация на атомном уровне.

Наиболее полно колебательная потенциальная энергия характеризуется суммой энергий растяжения связи $\left(\mathrm{E}^{\rho}\right)$, угловых изменений между соседними связями $\left(\mathrm{E}^{\theta}\right)$, энергией нековалентных взаимодействий $\left(\mathrm{E}^{\mathrm{VdW}}\right)$, энергией выхода связей из плоскости, кручением $\left(\mathrm{E}^{\text {tor }}\right)$, энергией изгиба плоскости образованной изменением $\pi$-электронной плотности $\left(\mathrm{E}^{\omega}\right)$.

$$
\mathrm{E}=\sum_{\substack{\text { связи } \\ \text { В моделирование нанотрубок лишь первые }}} \mathrm{E}^{\rho}+\sum_{\text {связи }} \mathrm{E}^{\theta}+\sum_{\text {связи }} \mathrm{E}^{\text {tor }}+\sum_{\text {связи }} \mathrm{E}^{\omega}+\sum_{\text {сяз }} \mathrm{E}^{\mathrm{VdW}}
$$
две суммы оказываются существенными. Энергия кручения оказывается весьма малой и ею можно пренебречь. Энергия изгиба $\mathrm{E}^{\omega}$ оказывается существенной при больших деформациях с изгибом. В случае если ее не принимать во внимание характер решения задач остается схожим с расчетами молекулярной динамики. В случае небольших изгибов $\mathrm{E}^{\omega}$ не сильно меняет значение. Лишь изменение потенциальной энергии является значимым.

При перестраивании дискретной модели в стержневую необходимо взять во внимание все значимые парные взаимодействия в структуре, и соотнести их с эквивалентными стержнями, тем самым заменив их.

Энергия деформации стержня оказывается равной энергии растяжения ковалентной связи $\mathrm{E}^{\rho}$, соединяющих пару атомов:

$$
\mathrm{E}^{\rho}=\mathrm{K}^{\rho}(\rho-\mathrm{P})^{2}
$$

В данной формуле $\mathrm{P}$ означает начальное межатомное расстояние, а $\rho$ уже деформированное.

$\mathrm{K}^{\rho}$ вычисляют из системы двух атомов вычислением преобразованием энергии системы, находящейся состояний преобразования. Этот 


\begin{tabular}{l|lr|ll|ll} 
& ISRA (India) & $=\mathbf{1 . 3 4 4}$ & SIS (USA) & $=\mathbf{0 . 9 1 2}$ & ICV (Poland) & $=\mathbf{6 . 6 3 0}$ \\
Impact Factor: & ISI (Dubai, UAE) $=\mathbf{0 . 8 2 9}$ & PUHI (Russia) & $=\mathbf{0 . 2 3 4}$ & PIF (India) & $=\mathbf{1 . 9 4 0}$ \\
& GIF (Australia) & $=\mathbf{0 . 5 6 4}$ & ESJI (KZ) & $=\mathbf{1 . 0 4 2}$ & IBI (India) & $\mathbf{4 . 2 6 0}$ \\
& JIF & $\mathbf{1 . 5 0 0}$ & SJIF (Morocco) & $\mathbf{2 . 0 3 1}$ & & \\
\hline
\end{tabular}

коэффициент характеризуется выбранным силовым полем, а так же формой вычисляемого потенциала.

$\mathrm{E}^{\theta}$ представляет собой энергию преобразования угла между соседними связями атома. С принятием во внимание валентности, а так же гибридизации и взаимодействия типов его соседей которые зависят от изменения угла:

$$
\mathrm{E}^{\theta}=\mathrm{K}^{\theta}(\theta-\Theta)^{2}
$$

В данной формуле начальное значение угла обозначается $\Theta$, а деформированное соответственно $\theta$.

В стержневой модели изменения углов между стержнями не оказывает никакое воздействие на энергию деформации, энергия деформации имеет зависимость лишь от деформации самих стержней.

На рисунке 2 представлены атомы образующие треугольник

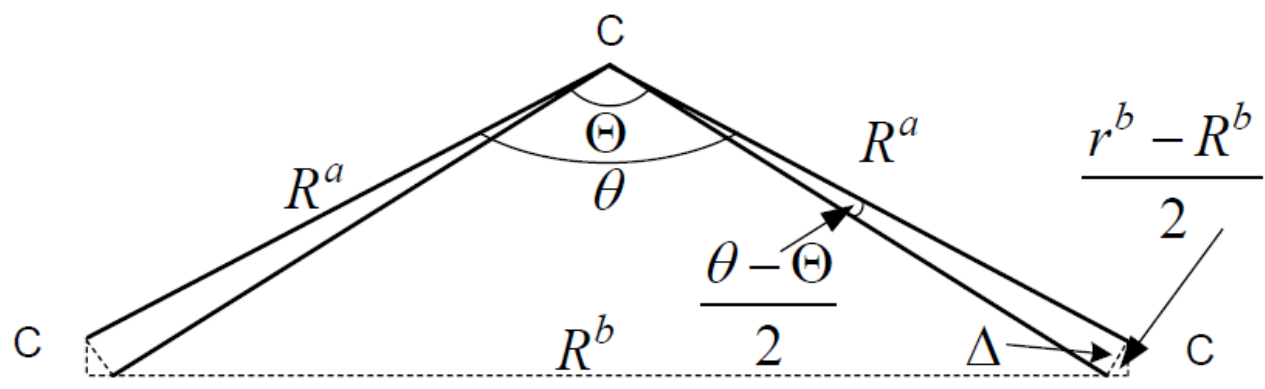

Рисунок 2 - Атомы формирующие треугольник.

Вычислим энергию деформации стержня с поперечным сечением А и объемом V.

Далее вычислим энергию упругой деформации системы, имеющей произвольную стержневую систему, вычисляем и модуль Юнга для двух типов стержней.

$$
\begin{gathered}
\mathrm{R}^{\mathrm{a}} \cos \left(\frac{\pi}{6}\right)=\frac{\mathrm{R}^{\mathrm{b}}}{2}, \quad \mathrm{R}^{\mathrm{a}} \frac{\sqrt{3}}{2}=\frac{\mathrm{R}^{\mathrm{b}}}{2}, \quad\left(\mathrm{R}^{\mathrm{a}}\right)^{2}=\frac{1}{3}\left(\mathrm{R}^{\mathrm{b}}\right)^{2} \\
\frac{4 \cdot 3 \mathrm{~K}^{\theta}}{\left(\mathrm{R}^{\mathrm{b}}\right)^{2}}=\frac{\mathrm{A}^{\mathrm{b}} \mathrm{Y}^{\mathrm{b}}}{2 \mathrm{R}^{\mathrm{b}}}, \quad \frac{24 \mathrm{~K}^{\theta}}{\mathrm{R}^{\mathrm{b}}}=\frac{\mathrm{A}^{\mathrm{b}} \mathrm{Y}^{\mathrm{b}}}{1}, \quad \mathrm{~K}^{\theta}=\frac{\mathrm{R}^{\mathrm{b}} \mathrm{A}^{\mathrm{b}} \mathrm{Y}^{\mathrm{b}}}{24} \\
\mathrm{Y}^{\mathrm{b}}=\frac{24 \mathrm{~K}^{\theta}}{\mathrm{R}^{\mathrm{b}} \mathrm{A}^{\mathrm{b}}}
\end{gathered}
$$

Межатомное расстояния $\mathrm{R}^{\mathrm{a}}$ и $\mathrm{R}^{\mathrm{b}}$ углерода в графите получены, а $\mathrm{K}^{\rho}$ и $\mathrm{K}^{\theta}$ описывают параметры силового поля. В случае если $\mathrm{A}^{\mathrm{a}}=\mathrm{A}^{\mathrm{b}}$ $=$ А возможно вычислить модули Юнга стержней.

Силовые константы примут значения:

$$
\begin{gathered}
\mathrm{K}^{\rho}=46900 \frac{\text { ккал }}{\text { моль } \cdot \mathrm{HM}^{2}} \\
\mathrm{~K}^{\theta}=63 \frac{\text { ккал }}{\text { моль } \cdot \text { рад }^{2}}
\end{gathered}
$$

В сравнении с длиной межатомных связей радиус стержней ничтожен и имеет значение 0.01 нм. Радиус таких стержней не оказывает влияния на решение. В моделях такие стержни играют роль пружины с жесткостью 163 Н/м для первого типа стержней. 9ТПа

Зная это, вычислим: $\mathrm{Y}^{\mathrm{a}}=295 \mathrm{TПа}, \mathrm{Y}^{\mathrm{b}}=$

Вычисление значения для третьего типа, которые описывают взаимодействие противолежащих атомов шестиугольников, не имеет смысла в виду отличия модуля Юнга первых двух более чем в 30 раз.

Для установления соответствия закона Вандер-Ваальса к гармоническому вычислим модуль Юнга присущий нековалентным взаимодействиям углерода. Полученное значение (500МПа) оказалось в 600 раз меньше $\mathrm{Y}^{\mathrm{a}}$.

Модель расположения атомов представлена на рисунке 3 . 


\begin{tabular}{l|lrl|l|ll} 
& ISRA (India) & $=\mathbf{1 . 3 4 4}$ & SIS (USA) & $=\mathbf{0 . 9 1 2}$ & ICV (Poland) & $=\mathbf{6 . 6 3 0}$ \\
Impact Factor: & ISI (Dubai, UAE) $=\mathbf{0 . 8 2 9}$ & PUHU (Russia) $=\mathbf{0 . 2 3 4}$ & PIF (India) & $=\mathbf{1 . 9 4 0}$ \\
& GIF (Australia) & $\mathbf{0 . 5 6 4}$ & ESJI (KZ) & $=\mathbf{1 . 0 4 2}$ & IBI (India) & $\mathbf{4 . 2 6 0}$
\end{tabular}

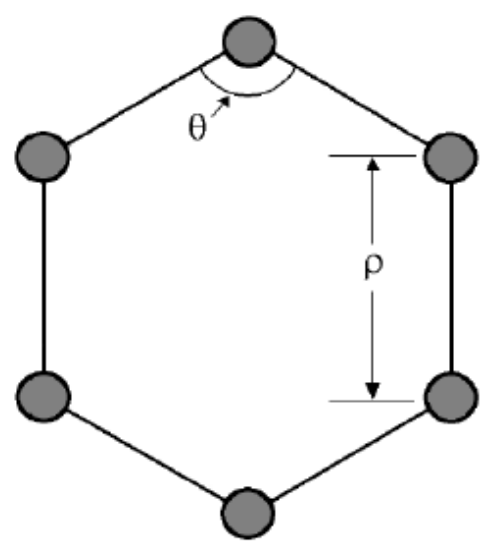

Химические

связи

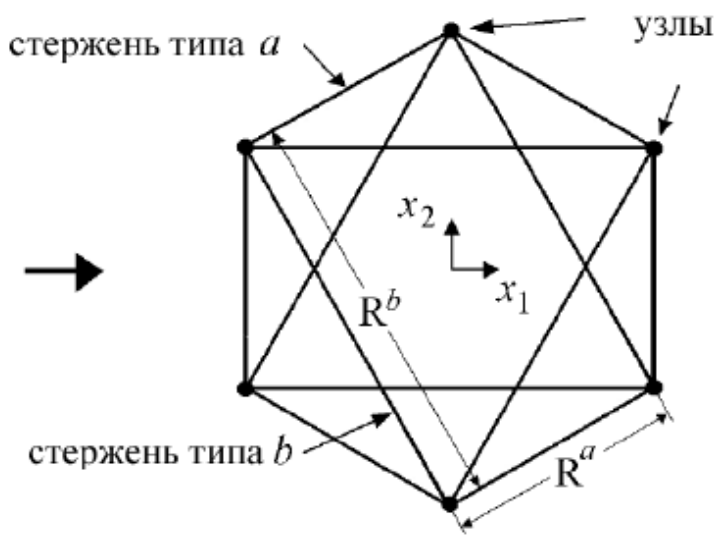

Стержневая модель с точечными шарнирами

Рисунок 3 - Модель расположения атомов.

При принятии во внимание парные взаимодействия в структуре и замене их соразмерными стержнями возможно перейти с дискретной к стержневой модели.

Энергии деформации стержня между парой атомов и энергией растяжения ковалентной связи $\mathrm{E}^{\rho}$ оказываются равными.

$$
\mathrm{E}^{\rho}=\mathrm{K}^{\rho}\left(\mathrm{r}^{\mathrm{a}}-\mathrm{R}^{\mathrm{a}}\right)^{2}
$$

$\mathrm{R}^{\mathrm{a}}$ и $\mathrm{r}^{\mathrm{a}}$ описывают исходное и соответственно деформированное межатомное расстояние. При выводе системы из равновесия и вычисление изменений энергии системы состоящей из двух атомов получим значение $\mathrm{K}^{\rho}$ для выбранных атомов. Характеристикой атома является преобразования угла между соседними связями атома $\mathrm{E}^{\theta}$

Завися от изменения угла и принимая во внимание соседнии взаимодействия их возможно вычислить по формуле $\mathrm{E}^{\theta}=\mathrm{K}^{\theta}(\theta-\Theta)^{2}$, переменные $\Theta$ обозначает начальное, а переменная $\theta$ обозначает значение угла но уже деформированного.

В стержневой модели деформационная энергия зависит лишь от деформаций стержней и не как не зависит угловых изменений.

Зависимость $E^{\theta}$ относительно угла возможно заменить на $\mathrm{E}^{\theta}==4 \mathrm{~K}^{\theta}(\rho-\mathrm{P})^{2} / \mathrm{P}^{2}$.

Покрывая всю гексагональную плоскость стержнями двух типов модель во время деформации проявляет себя подобно модели молекулярной динамики. Данный подход построения стержневых систем эквивалентных атомным удобен для применения в моделировании наноструктурных объектов и различных материалов имеющих в основе наночастицы.

Энергия накопленная во время изгиба графеновой плоскости связанна с изменением $\pi$ орбитальной электронной плотности.

Имея возможность представления нанотрубок в виде стержневых моделей можно начать исследовать степень ее изотропии. В виду того, что предыдущей формой нанотрубки является графеновая плоскость обладающая на макро уровне изотропией упругих свойств несмотря на свою гексагональность.

Имеются физические задачи, решение которых не требуют моделирования абсолютно всех свойств нанотрубок, их полое представление в форме цилиндра с конечной толщиной. В таких задачах достаточно представлении нанотрубок в форме сплошных цилиндров. Такими задачами будут укрепление композиционных материалов волокнами нанотрубок для упрочнения композита. В таких задачах нанотрубка это сплошной цилиндр, сформированный из анизотропного материала, так называемого нановолокна. Степень изотропии в таких материалах свойственна нанотрубкам, изотропии свойств в плоскости, которая расположена поперек оси трубки.

Система нано масштабных параллельно расположенных волокон, представленная на рисунке 4 представляет собой трансверсально изотропную среду.

В связи с тем, что нановолокно считаем трансверсально изотропной средой, тензор упругих констант для таких сред $\mathrm{C}_{\mathrm{ijkl}}$ возможно вычислить и экспериментально. 


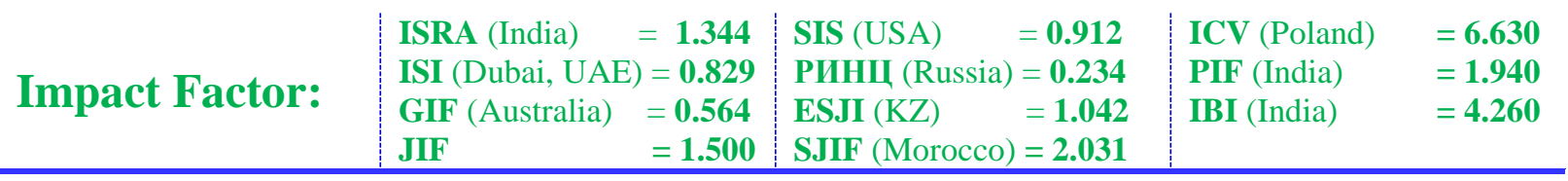

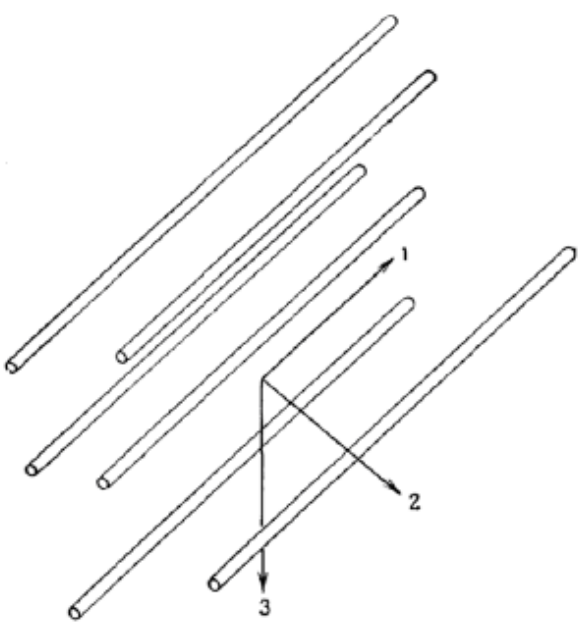

Рисунок 4 - Система нано масштабных параллельно расположенных волокон.

Наиболее легким объектом для моделирования является графеновая плоскость или ее фрагменты. Такие фрагменты и плоскости исследуются энергетическим методом. Так же изучалось влияние вырезания фрагмента на модуль Юнга, исследовались два различных случая для образцов при разных углах хиральности $\left(\alpha=0^{\circ}\right.$ и $\left.30^{\circ}\right)$, эти фрагменты характеризуются векторами $\underset{C}{\rightarrow}$ и $\underset{L}{\rightarrow}$, либо другим способом это индексами $(\mathrm{m}, \mathrm{n}): \mathrm{k}$.

В случае использования нанотрубок в роли упрочняющего наполнителя в полимерных композитах, атомы формирующие нанотрубку так же вступают в связи с атомами находящимися вокруг них в полимерной матрице. Взаимодействия атомов могут быть двух видов, таких как ковалентные и Ван-дер-Ваальсовые. Модели нанотрубок, в особенности стержневая, позволяет оценивать внешние влияния на связи и упругие свойства волокон.
Для облегчения вычислений принята модель в которой все атомы нановолокон связаны с матрицей. Хотя такое взаимодействие и не реализуемо на данный момент, легче всего начать c него. Моделирование внешних влияний на стержни проводились благодаря моделям стержней типа №3 построенных по нормали из каждого атома с поверхности трубки. Их длина строилась исходила из межатомного расстояния. Моделирование такой нанотрубки в единичном варианте представлены на рисунке 5. Один конец такой нанотрубки закреплялся и далее она растягивается подобно экспериментам из определения модуля Юнга. В таких вариантах каждый раз выбирается различный модуль юнга для Зго вида стержней. В данном исследовании учитываются ковалентные взаимодействия на поверхностях нанотрубок с матрицей в отличии от подобных исследованиях [2], где они не учитывались.

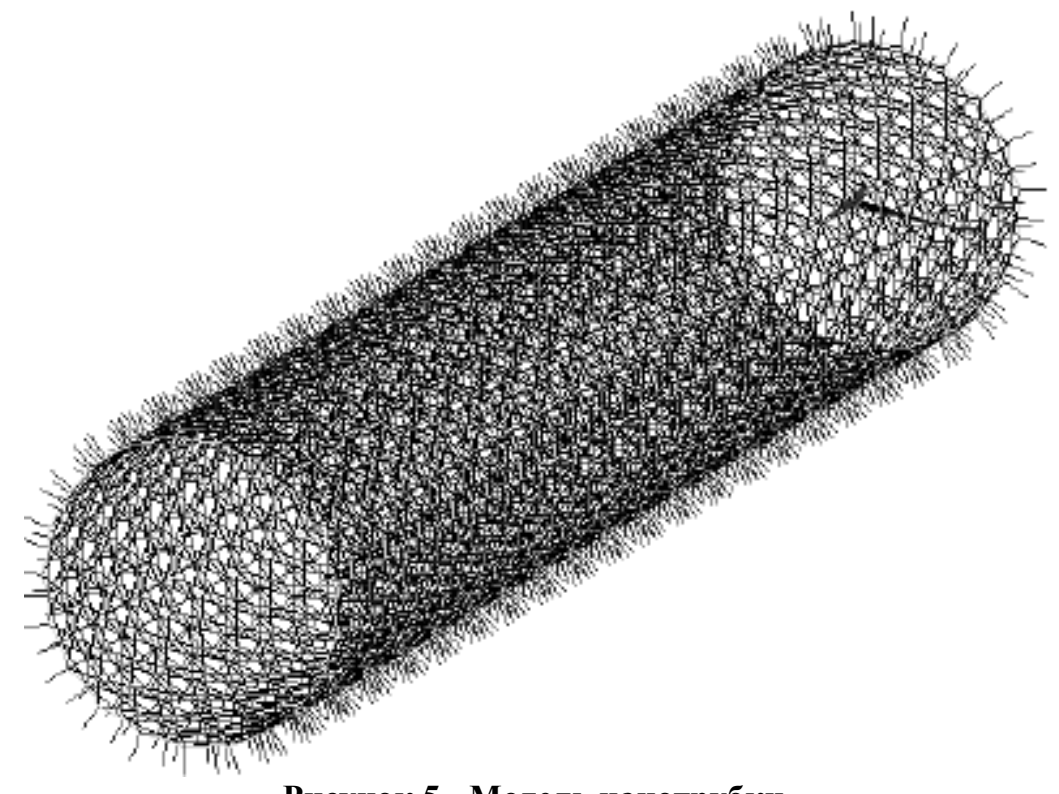

Рисунок 5 - Модель нанотрубки. 
Нековалентное

взаимодействие позволяющее взаимодействовать для каждой нанотрубки взимодействовать ее атомов с контактирующими с ними атомами других элементов входящих в состав композита не дает ожидаемого эффекта, тем самым не происходит перераспределения внешних воздействий на матрицу.

Результатом теоретических исследований по улучшению упругих свойств материалов является вывод, что наполнение материалов нанотрубками и при формировании крепких ковалентных связей между наполнителем и матрицей дают значимые результаты. И формирование лишь Ван дер Ваальсовых взаимодействий между ними становится малы для получения нужных характеристик материалов.

Исследование потери устойчивости и тем самым изменение формы так же важный аспект исследований.

Преобразование формы и поведения нанотрубок при сжатиях один важнейших экспериментов также проводимым многими, к примеру в [3]).

Мы исследуем осевое ее сжатие. К моделям с заданными граничными условиями применялись конечные перемещения с сжимающими силами для узлов.

Нижний и верхний шестиугольник из атомов в нанотрубке закреплялись не подвижно к перпендикулярным осям относительно нанотрубки.

При изменении параметров линейных размеров для нанотрубок их устойчивость к сжатию близится к классическим сжатиям упругих стержней(свойственно для тонких и длинных нанотрубок), а для коротких трубок свойственны устойчивости как для оболочек.

В нанотрубках и графеновых плоскостях при формировании структуры побочно образуются и дефекты, для гексагональных плоскостей с ковалентными связями(не параллельных осям растяжений) дефекты представляются энергетически выгодными. Нанотрубки не всех типов подвержены формированию дефектов, тип «кресло» наиболее подвержен. Для гексагональных плоскостей это тоже свойственно так как нанотрубки их и образуют. При угле хиральности равном 30 градусов формирование дефектов наиболее ярко проявляется при растяжении. Исследование таких образцов наиболее перспективно.

Проблемы моделирования механических свойств материалов как и раньше актуальны, особенно с учетом их структуры в моделях. Решение данных проблем привод к учету большого количества зависимостей материала от его макроскопических характеристик деформирования, а также его прочностных характеристик и в особой степени его сопротивления разрушению композитного материала в зависимости от параметров структуры.

Рассчитав данные параметры зависимостей структуры, есть возможность оптимизировать и рассчитать характеристики композитных материалов, создаваемых конструкций на их основе и многое другое.

Применяемый дискретно континуальный подход позволяет упростить распространенные атомистические методы, и существенно облегчить моделирование, особенно в количестве проводимых вычислений, позволяет моделировать многомасштабные структуры и их поведение, деформирование на нано, макро и мезомасштабах.

Значимость и в большей мере трудность реализации поставленных в рамках данной диссертации задач сфокусирована в существующих методах расчетов атомных систем. К таким методам относится метод молекулярной динамики. Он характеризуется ресурсоемкостью и сложностью в анализе при решении задач.

\section{References:}

1. Coleman JN, Khan U, Blau WJ, Gun'ko YK (2006) Small but strong: A review of the mechanical properties of carbon nanotubepolymer composites. Carbon. 2006. V. 44. No9. pp. 1624-1652.

2. Aldoshin SM, Badamshina ER, Kablov EN (2008) Polimernye nanokompozity -novoe pokolenie polimernykh materialov $\mathrm{s}$ povyshennymi ekspluatatsionnymi kharakteristikami. Sb. trudov. Mezhdunar. foruma po nanotekhnologiyam «Rusnanotech 08». M.: ROSNANO, 2008. T.1. pp.385-386.

3. Tarasov VA, Stepanishchev NA (2010) Primenenie nanotekhnologiy dlya uprochneniya poliefirnoy matritsy kompozitsionnogo materiala. Vestnik MGTU im N E. Baumana No 2010, pp. 25-36.

4. (1975) Sb.: Kvantovye kristally (perevody) / Pod red. Vonsovskogo S.V. M.: Mir.1975. 280 p. 


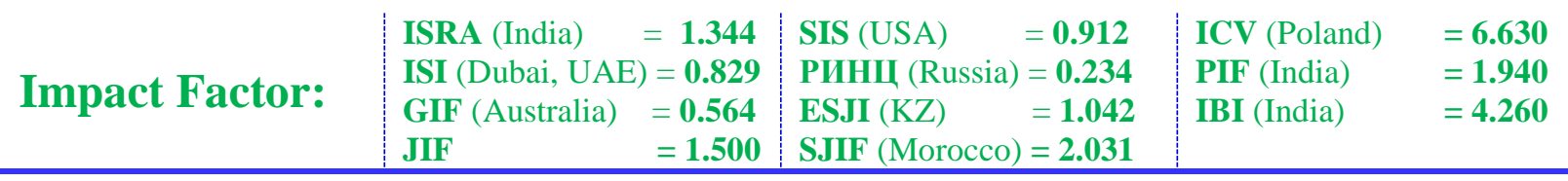

5. Johnson RA (1973) Empirical potentials and their use in the calculation of energies of point defects in metals // J. Phys. F: Metal Physics. 1973. V.3. N. 2. pp. 295-321.

6. Malamatov AK, Kozlov GV, Mikitaev MA (2006) Mekhanizmy uprochneniya polimernykh nanokompozi-tov. - Moskva: Izd-vo RKhTU im. Mendeleeva, 2006. $-240 \mathrm{p}$.

7. Budtov VP (1992) Fizicheskaya khimiya rastvorov polimerov. - Sankt-Peterburg: Khimiya, 1992. - 384 p.

8. Burya AI, Kozlov GV, Rula IV (2004) Obobshchennaya metodika otsenki soderzhaniya mezhfaznykh oblastey $\mathrm{V}$ polimernykh kompozitakh // Novosti nauki Pridneprov'ya. - 2004. - No 3. - pp. 8-11.

9. Malamatov AK, Kozlov GV, Antipov EM, Mikitaev MA (2006) Mekhanizm formirovaniya mezhfaznykh sloev $\mathrm{V}$ polimernykh nanokompozitakh // Perspektiv. materialy. - 2006. - No 5. - pp. 54-58.

10. (1988) Spravochnik po kompozitsionnym materialam/Pod red. Dzh. Lyubina, per. s angl. Pod red. A. B. Gellera, M.M. Gel'monta Pod red. B. E. Gellera. M: Mashinostroenie, 1988. $448 \mathrm{p}$.

11. Nil'sen L (1978) Mekhanicheskie svoystva polimerov i polimernykh kompozitsiy. M: Khimiya, 1978. 310 p. 